\title{
Trends in Spitzer Secondary Eclipses
}

\author{
Nicole L. Wallack ${ }^{1}$ (D), Heather A. Knutson ${ }^{1}$, and Drake Deming ${ }^{2}$ \\ ${ }^{1}$ Division of Geological \& Planetary Sciences, California Institute of Technology, Pasadena, CA 91125, USA; nwallack@ caltech.edu \\ ${ }^{2}$ Department of Astronomy, University of Maryland at College Park, College Park, MD 20742, USA \\ Received 2020 February 27; revised 2021 January 1; accepted 2021 January 12; published 2021 June 29
}

\begin{abstract}
It is well established that the magnitude of the incident stellar flux is the single most important factor in determining the day-night temperature gradients and atmospheric chemistries of short-period gas giant planets. However, it is likely that other factors, such as planet-to-planet variations in atmospheric metallicity, C/O ratio, and cloud properties, also contribute to the observed diversity of infrared spectra for this population of planets. In this study, we present new 3.6 and $4.5 \mu \mathrm{m}$ secondary eclipse measurements for five transiting gas giant planets: HAT-P-5b, HAT-P-38b, WASP-7b, WASP-72b, and WASP-127b. We detect eclipses in at least one bandpass for all five planets and confirm circular orbits for all planets except WASP-7b, which shows evidence for a nonzero eccentricity. Building on the work of Garhart et al., we place these new planets into a broader context by comparing them with the sample of all planets with measured Spitzer secondary eclipses. We find that incident flux is the single most important factor for determining the atmospheric chemistry and circulation patterns of shortperiod gas giant planets. Although we might also expect surface gravity and host star metallicity to play a secondary role, we find no evidence for correlations with either of these two variables.
\end{abstract}

Unified Astronomy Thesaurus concepts: Exoplanet atmospheres (487); Exoplanet atmospheric composition (2021); Exoplanets (498); Broad band photometry (184); Infrared photometry (792)

\section{Introduction}

For short-period gas giant planets with hydrogen-rich envelopes, the amount of incident flux received from the star is predicted to be the primary factor that determines the shape of their observed dayside emission spectra (e.g., Burrows et al. 2008; Fortney et al. 2008). We expect these short-period planets to be tidally locked, where the efficiency of day-night circulation varies as a function of the incident flux (e.g., Komacek \& Showman 2016). This atmospheric circulation in turn determines the temperature of the upper region of the dayside atmosphere, which sets the equilibrium chemistry and corresponding atmospheric composition (e.g., Heng \& Marley 2018). Although photochemistry and mixing from the nightside and deep interior can alter this default chemistry, these effects are predicted to be below the sensitivity of current observations for the majority of planets observed to date (e.g., Moses 2014). Condensate clouds can also alter the observed atmospheric properties of these planets, but the effects of these clouds are expected to be less pronounced for dayside thermal emission spectra than for transmission spectroscopy (e.g., Fortney 2005).

Observations of the secondary eclipse, when the planet passes behind its host star, allow us to probe the thermal emission spectra of transiting gas giant planets. This has enabled detailed studies of a handful of planets (e.g., Brogi et al. 2017; Morley et al. 2017; Kreidberg et al. 2018), but there are relatively few planets with such extensive secondary eclipse data sets. If we broaden our focus to planets with just a few broadband photometric measurements from Spitzer, we can search for broader population-level trends. Previous studies of dayside emission spectra confirm that hotter planets do indeed have less efficient heat redistribution (Schwartz \& Cowan 2015; Garhart et al. 2020), in good agreement with predictions from atmospheric circulation models (Perez-Becker \& Showman 2013; Komacek \& Showman 2016). Garhart et al. (2020) additionally found evidence for a systematic shift in the 3.6-4.5 $\mu \mathrm{m}$ spectral slopes of these planets as a function of incident flux, which suggests that the atmospheric chemistries and pressure-temperature profiles of these planets also vary as a function of the irradiation. However, this study found that neither of the two most commonly utilized model atmosphere grids was able to accurately predict the increase in the observed ratio of 3.6 and $4.5 \mu \mathrm{m}$ brightness temperatures with increasing equilibrium temperature, suggesting that these models can be further improved.

To date, most published population-level studies of transiting gas giant planet emission spectra have focused on searching for correlations with the incident flux (e.g., Cowan \& Agol 2011; Schwartz \& Cowan 2015; Schwartz et al. 2017; Baxter et al. 2020; Garhart et al. 2020). However, we expect that planets with the same incident flux levels might nonetheless possess distinct thermal spectra if they have different atmospheric metallicities and/or surface gravities, both of which can alter their atmospheric chemistries, circulation patterns, and cloud properties. In a previous study (Wallack et al. 2019), we focused on the subpopulation of planets cooler than $\sim 1000 \mathrm{~K}$, which are expected to undergo a particularly distinct shift in atmospheric chemistry as a function of atmospheric metallicity and $\mathrm{C} / \mathrm{O}$ ratio (e.g., Moses et al. 2013; Drummond et al. 2018). In this study, we broaden our focus to the full sample of transiting gas giant planets with Spitzer secondary eclipse detections in order to determine whether or not there are additional parameters beyond incident flux that might help to explain the observed diversity of dayside emission spectra. These same factors might also provide new insights into how to modify standard atmosphere model grids in order to better match the observed trends in spectral shape as a function of incident flux.

Garhart et al. (2020) presented a uniform analysis of 3.6 and $4.5 \mu \mathrm{m}$ Spitzer observations of 36 transiting hot Jupiters. We use 31 of these observations (those with detections above $2.5 \sigma$ ) and expand on this sample by leveraging an additional 42 planets with Spitzer 3.6 and $4.5 \mu \mathrm{m}$ secondary eclipse detections (above $2.5 \sigma$ ) 
Table 1

System Properties for New Planets in This Study

\begin{tabular}{|c|c|c|c|c|c|}
\hline & HAT-P-5b & HAT-P-38b & WASP-7b & WASP-72b & WASP-127b \\
\hline$T_{*}(\mathrm{~K})$ & $5960 \pm 100$ & $5330 \pm 100$ & $6520 \pm 70$ & $6250 \pm 100$ & $5750 \pm 100$ \\
\hline$[\mathrm{Fe} / \mathrm{H}]_{*}$ & $0.24 \pm 0.15$ & $0.06 \pm 0.10$ & $0.00 \pm 0.10$ & $-0.06 \pm 0.09$ & $-0.18 \pm 0.06$ \\
\hline Mass $\left(\mathbf{M}_{\text {Jup }}\right)$ & $1.06 \pm 0.11$ & $0.267 \pm 0.020$ & $0.98 \pm 0.13$ & $1.461_{-0.056}^{+0.059}$ & $0.165_{-0.017}^{+0.021}$ \\
\hline Radius ( $\mathbf{R}_{J u p}$ ) & $1.252 \pm 0.043$ & $0.825_{-0.063}^{+0.092}$ & $1.374 \pm 0.094$ & $1.27 \pm 0.20$ & $1.311_{-0.029}^{+0.025}$ \\
\hline$T_{\text {equ }}(\mathrm{K})^{\mathrm{a}}$ & $1517 \pm 37$ & $1080_{-45}^{+60}$ & $1530 \pm 50$ & $2204_{-115}^{+139}$ & $1404 \pm 29$ \\
\hline$e^{\mathrm{b}}$ & $<0.072(<0.18)$ & $<0.055(<0.17)$ & $<0.049(<0.11)$ & $<0.017(<0.038)$ & 0 \\
\hline$\omega(\operatorname{deg})^{\mathrm{b}}$ & $\ldots$ & $\ldots$ & $\ldots$ & $\ldots$ & $\cdots$ \\
\hline Period (days) ${ }^{\mathrm{c}}$ & $2.78847360(52)$ & $4.640382(32)$ & $4.9546416(35)$ & $2.2167421(81)$ & $4.17807015(57)$ \\
\hline$T_{c}(\mathrm{BJD}-2,450,000)^{\mathrm{c}}$ & $5,432.45510(10)$ & $5,863.12034(35)$ & $5,446.63493(30)$ & $5,583.6528(21)$ & $7,248.741276(68)$ \\
\hline References & $1,2,3,4,5$ & $1,5,6$ & $1,5,7,8$ & $1,5,9$ & $5,10,11$ \\
\hline
\end{tabular}

Notes.

${ }^{\text {a }}$ Calculated assuming planetwide heat circulation and zero albedo.

b The orbital eccentricity $e$ and longitude of periapse $\omega$ are derived from fits to radial velocity data.

${ }^{c}$ Uncertainties on the last two digits are in parentheses.

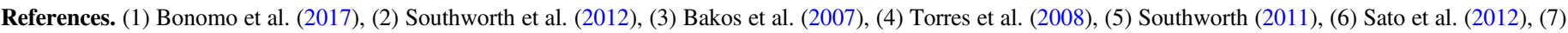
Albrecht et al. (2012), (8) Hellier et al. (2009), (9) Gillon et al. (2013), (10) Lam et al. (2017), (11) Chen et al. (2018).

from the literature, as well as adding secondary eclipse measurements for three of our five new planets: HAT-P-5b (Bakos et al. 2007), WASP-7b (Hellier et al. 2009), and WASP127b (Lam et al. 2017). HAT-P-38b (Sato et al. 2012) and WASP-72b (Gillon et al. 2013) do not have detections in both bandpasses. With this newly expanded sample, we proceed to revisit previously established correlations between spectral shape and incident flux and search for additional correlations with stellar metallicity and surface gravity. In Section 2, we describe our photometric extraction and model fits. In Section 3, we present new Spitzer secondary eclipse measurements of five new planets, and in Section 4, we add these new planets to the published Spitzer secondary eclipse measurements and investigate trends in the thermal emission spectra of the population of short-period gas giant planets.

\section{Observations and Data Analysis}

We obtained one secondary eclipse each in the IRAC 3.6 and $4.5 \mu \mathrm{m}$ bands (Fazio et al. 2004) for HAT-P-5b (PID: 60021), WASP-7b (PID: 60021), WASP-72b (PID: 10102), and WASP127b (PID: 13044) and two visits in each band for HAT-P-38b (PID: 12085). Aside from HAT-P-5b, all of these data were taken in the $32 \times 32$ pixel subarray mode with an initial 30 minute observation to allow for settling of the telescope followed by a peak-up pointing adjustment prior to the start of the science observation (Ingalls et al. 2012). HAT-P-5b and WASP-7b are the oldest data sets in this study and were observed before the peak-up pointing mode was fully implemented. Additionally, HAT-P-5b was observed in full array mode. See Tables 1 and 2 for additional details.

We utilize the standard Basic Calibrated Data images for our analysis and extract photometric fluxes as described in our previous studies (i.e., Wallack et al. 2019). In brief, we first calculate the $\mathrm{BJD}_{\mathrm{UTC}}$ mid-exposure times for each image, then estimate the sky background in each image by masking out a circular region with a radius of 15 pixels centered on the position of the star, iteratively trimming $3 \sigma$ outliers, and fitting a Gaussian function to a histogram of the remainder of the pixels. We utilize flux-weighted centroiding (e.g., Knutson et al. 2008; Deming et al. 2015) with a circular aperture to determine the location of the star on the array, considering aperture radii ranging between 2.5 and 4.0 pixels in 0.5 pixel steps, and optimize our choice of aperture as described below. We use the aper routine in the DAOPhot package (Stetson 1987) to extract the total flux in a circular aperture centered on the position of the star, considering aperture sizes ranging from 2.0 to 3.0 pixels in steps of 0.1 pixel and 3.0 to 5.0 pixels in steps of 0.5 pixel.

In order to mitigate the ramp-like behavior present at early times in some of the visits, we trim up to $2 \mathrm{hr}$ of data from the beginning of each time series. We find that binning our data prior to fitting reduces the amount of time-correlated noise in the residuals (see Deming et al. 2015 and Kammer et al. 2015 for more details). In order to determine the optimal combination of flux-weighted centroiding aperture, photometric aperture, trim duration, and bin size for each visit, we fit a combined instrumental and astrophysical model to each version of the photometry and calculate the standard deviation of the residuals as a function of bin size stepping in powers of two (see Kammer et al. 2015 for further details). We then calculate the leastsquares difference between the measured standard deviation of the residuals and the predicted photon noise limit in each bin, which decreases as the square root of the number of points. We then select the photometric and centroiding apertures, trim duration, and bin size that minimizes this least-squares difference (i.e., the one that is closest to the photon noise at all measured timescales) for use in our subsequent analysis.

Our model for each visit consists of a secondary eclipse light curve and an instrumental noise model, which we fit simultaneously. We calculate our eclipse model using the batman package (Mandel \& Agol 2002; Kreidberg 2015), where we fix the planet-star radius ratio, orbital inclination, and ratio of the orbital semimajor axis to the stellar radius $\left(a / R_{*}\right)$ to the published values for each planet (see Table 1 for references) and allow the eclipse depth and time to vary as free parameters. Due to the fact that the orbital parameters are often more precisely measured from transit light curves than from secondary eclipse light curves, we are justified in fixing the orbital parameters to those measured from transit light curves instead of letting these parameters vary in our fits.

The dominant instrumental noise source for Spitzer timeseries photometry is intrapixel sensitivity variations (Charbonneau et al. 2005; Reach et al. 2005; Morales-Calderon et al. 2006), which cause the apparent flux from the star to vary as a 
Table 2

Spitzer Observation Details

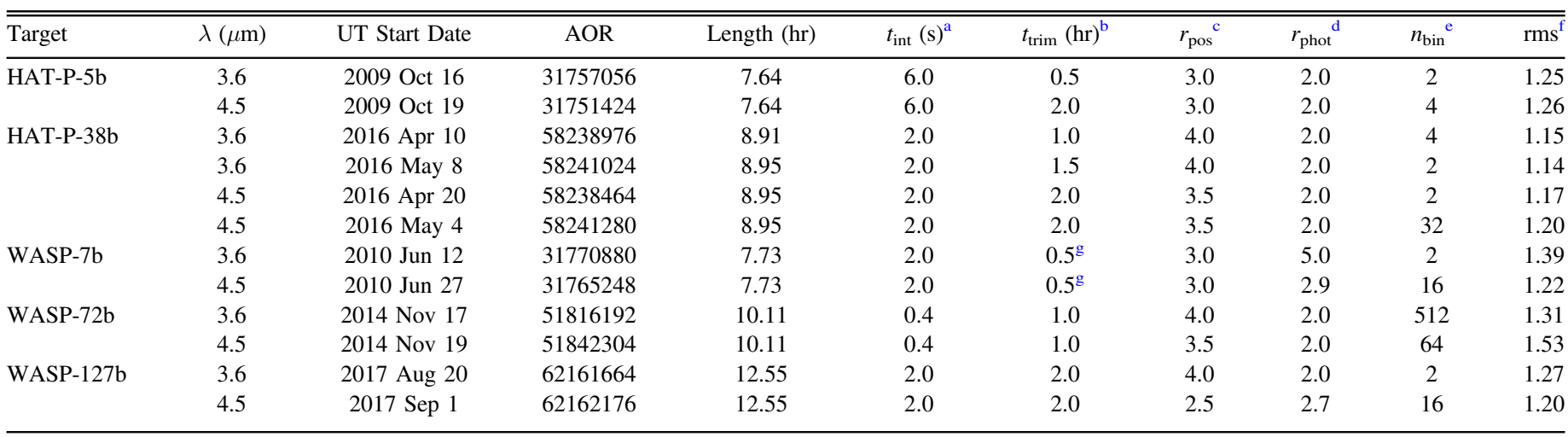

Notes.

${ }^{\mathrm{a}}$ Integration time.

b Initial trim duration.

${ }^{c}$ Radius of the aperture (in pixels) used to determine the location of the star on the array.

${ }^{\mathrm{d}}$ Radius of the aperture (in pixels) used for the photometry.

e Bin size used for fits.

${ }^{\mathrm{f}}$ Ratio of measured rms to photon noise limit.

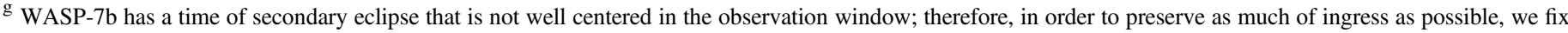
the trim duration to 30 minutes (see Section 2 for more details).

function of its position on the pixel. We model this effect using the pixel-level decorrelation (PLD) method, which uses a linear combination of individual pixel-level light curves to account for trends due to variations in both the star's position on the array and the width of the stellar point-spread function (Deming et al. 2015). As in Deming et al. (2015), we utilize a $3 \times 3$ grid of pixels centered on the location of the star and remove astrophysical flux variations in each $3 \times 3$ postage stamp by dividing by the summed flux across all nine pixels.

In the majority of our observations, we can fully account for additional time-dependent trends with the inclusion of a linear function of time once we have trimmed some data from the start of the observation. This is not true, however, for the $3.6 \mu \mathrm{m}$ observation of WASP-72b or the $3.6 \mu \mathrm{m}$ observation of WASP-7b. For the $3.6 \mu \mathrm{m}$ observation of WASP-72b, we obtain the best fit using an exponential function of time. Using this exponential reduces the Bayesian information criterion (BIC) by 12 . For the $3.6 \mu \mathrm{m}$ observation of WASP-7b, we obtain the best fit using a quadratic function of time ( $\triangle \mathrm{BIC}$ of 66). For all fits, we divide out our initial astrophysical model and use linear regression on the residuals to obtain an initial guess for the nine linear PLD coefficients in order to speed up convergence for these highly correlated parameters.

When optimizing our choice of photometry, we first fix the predicted time of eclipse to an orbital phase of 0.5 and run fits on each version of the photometry using a Levenberg-Marquardt minimization. We then select the optimal version of the photometry in each bandpass and carry out a simultaneous fit to all of the visits for a given planet, where we allow the orbital phase of the secondary eclipse to vary as a free parameter. We carry out these fits using the affine-invariant Markov Chain Monte Carlo (MCMC) ensemble sampler emcee (ForemanMackey et al. 2013, 2019), where we allow the secondary eclipse depth to vary independently in each bandpass but assume a common eclipse phase. We place uniform priors on all free parameters and allow the eclipse depths to take on negative values so that we do not bias our eclipse depth estimates. We utilize 60 walkers for our fits, which is enough to ensure adequate sampling of the model parameter space. We initialize these walkers in a tight cluster centered on the best-fit solution from a joint Levenberg-Marquardt minimization and carry out an initial burn-in with a length of 10,000 steps. We then discard this initial burn-in and carry out a subsequent fit with $10^{5}$ steps per chain. We then return to the original set of photometry options and repeat our optimization fixing the time of secondary eclipse to the median value from the MCMC chains. We adopt the resulting optimal photometry choices for each visit and rerun the MCMC for the joint fits.

We report the median values from our MCMC chains and the corresponding $1 \sigma$ uncertainties in Table 3 and show the raw photometry for each visit with the best-fit instrumental noise models from the joint fits overplotted in Figure 1. Normalized light curves for these visits with the best-fit eclipse light curves overplotted are shown in Figure 2. In Figure 3, we combine all visits for HAT-P-38b and show the averaged light curves for each bandpass. The standard deviations of the residuals as a function of bin size for all visits are shown in Figure 4.

We alter our fitting procedure for WASP-7b, as there appears to be substantial correlated noise (i.e., the residuals do not scale with $\sqrt{n}$ ) in the residuals of the $3.6 \mu$ m data (see the WASP-7b $3.6 \mu \mathrm{m}$ panel in Figure 4). To mitigate any biases in our bestfit parameters, we initially fit each of the channels for WASP$7 \mathrm{~b}$ independently. We find that the best-fit secondary eclipse phases for each channel are consistent at the $1 \sigma$ level, indicating that the correlated noise in the $3.6 \mu \mathrm{m}$ data is likely not biasing our time of secondary eclipse in that channel.

We find that both the 3.6 and $4.5 \mu \mathrm{m}$ data prefer an eclipse phase that is offset from the expected value for a circular orbit (see Section 3 for more details). As a result, the secondary eclipse is not centered in the observation but instead occurs $78.8_{-4.2}^{+5.0}$ minutes early. In order to preserve as much of ingress as possible, we only trim 30 minutes from the beginning of each observation for this planet, rather than considering a range of trim durations and optimizing to minimize the scatter in the residuals. We account for the effect of correlated noise on the 
Table 3

Best-fit Eclipse Parameters

\begin{tabular}{|c|c|c|c|c|c|c|}
\hline Target & Band $(\mu \mathrm{m})$ & Depth (ppm) & $T_{\text {Bright }}(\mathrm{K})$ & Time Offset (days) ${ }^{\mathrm{a}}$ & Center of Eclipse (Phase) & $e \cos (\omega)^{\mathrm{b}}$ \\
\hline \multirow[t]{2}{*}{ HAT-P-5b } & 3.6 & $908_{-201}^{+202}$ & $1485_{-118}^{+109}$ & $-0.0006_{-0.0022}^{+0.0024}$ & $0.4998_{-0.0008}^{+0.0009}$ & $-0.0003_{-0.0012}^{+0.0014}$ \\
\hline & 4.5 & $1508 \pm 266$ & $1567_{-121}^{+115}$ & & & \\
\hline HAT-P-38b & 3.6 & $698 \pm 189^{c}$ & $1503_{-150}^{+135}$ & $-0.0113_{-0.0042}^{+0.0067 \mathrm{e}}$ & $0.4976_{-0.0009}^{+0.0014}$ & $-0.0038_{-0.0014}^{+0.0023}$ \\
\hline \multirow[t]{2}{*}{ WASP-7b } & 3.6 & $714_{-190}^{+191 \mathrm{c}}$ & $1583_{-161}^{+147}$ & $-0.0547_{-0.0029}^{+0.0035}$ & $0.4890_{-0.0006}^{+0.0007}$ & $-0.0173_{-0.0009}^{+0.0011}$ \\
\hline & 4.5 & $725_{-106}^{+109}$ & $1393_{-82}^{+80}$ & & & \\
\hline WASP-72b & 3.6 & $<852^{\mathrm{c}, \mathrm{d}}$ & $<2265$ & $-0.0009_{-0.0071}^{+0.0054}$ & $0.4996_{-0.0032}^{+0.0024}$ & $-0.0006_{-0.0050}^{+0.0038}$ \\
\hline \multirow[t]{2}{*}{ WASP-127b } & 3.6 & $719 \pm 62$ & $1454_{-43}^{+42}$ & $0.0038_{-0.0015}^{+0.0013}$ & $0.5009_{-0.0004}^{+0.0003}$ & $0.0014_{-0.0006}^{+0.0005}$ \\
\hline & 4.5 & $910 \pm 69$ & $1373_{-41}^{+40}$ & & & \\
\hline
\end{tabular}

Notes.

a Time offset from predicted center of eclipse. We fit both channels with a common time of secondary eclipse.

b Computed using the approximation for a low-eccentricity orbit.

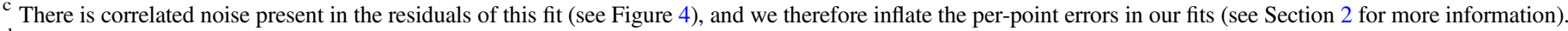

${ }^{\mathrm{d}}$ We report the $2 \sigma$ upper limit for the eclipse depth.

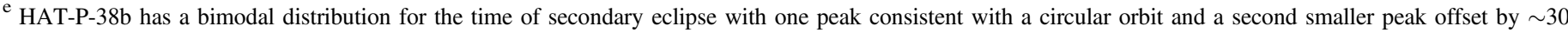
minutes. We report the eclipse depths and time for the solution that is consistent with a circular orbit; see Section 3 for more details.

$3.6 \mu \mathrm{m}$ eclipse depth uncertainty by inflating the per-point errors (which are generally left as a free parameter in our fits) by a factor that reflects how much the variance of the residuals deviates from the expected white-noise scaling (i.e., $1 / \sqrt{n}$, where $n$ is the number of points in each bin) at a characteristic timescale of 10 minutes (see Pont et al. (2006) and Lanotte et al. (2014) for more information). Because we are fitting binned light curves, we calculate this inflation factor as the amount of excess noise relative to the expected $\sqrt{n}$ scaling when we go from the binning timescale used in the fits to a binning timescale of 10 minutes (see Figure 4). We find that the resulting inflation factor is 2.1 for the $3.6 \mu \mathrm{m}$ WASP-7b observation. We then take our best-fit per-point error from the initial fit, multiply it by that factor, fix the per-point uncertainty to that value, and rerun our fit in order to obtain an updated eclipse depth and phase.

It is apparent in Figure 4 that several other observations also appear to have excess correlated noise. We calculate the inflation factor for each observation following the same process as described above and implement a new version of the fit with an inflated per-point uncertainty for visits with inflation factors larger than 1.5. We find that correlated noise exceeding this threshold is present in both the 3.6 and $4.5 \mu \mathrm{m}$ observations of WASP-72b (inflation factors of 1.5 and 2.0, respectively; see Figure 4) and both $3.6 \mu \mathrm{m}$ observations of HAT-P-38b (inflation factors of 1.7 and 1.5 for the first and second observations, respectively). For WASP-72b, we do not detect the eclipse in the $3.6 \mu \mathrm{m}$ bandpass, so we cannot compare the best-fit secondary eclipse phases from each channel in order to determine if this parameter is affected by correlated noise. However, the best-fit secondary eclipse phase from the $4.5 \mu \mathrm{m}$ fit agrees with the prediction for a circular orbit, and the alternative scenario (slightly eccentric orbit biased by correlated noise to appear circular) seems unlikely.

\section{Results}

We report the best-fit eclipse depths and times and their corresponding uncertainties in Table 3 . We detect the eclipse in both bandpasses with greater than $3 \sigma$ significance for HAT-P$5 b$, WASP-7b, and WASP-127b. For HAT-P-38b, we detect the eclipse at $3.6 \mu \mathrm{m}$ but not $4.5 \mu \mathrm{m}$, and for WASP-72b, we detect the eclipse at $4.5 \mu \mathrm{m}$ but not $3.6 \mu \mathrm{m}$. This allows us to place relatively tight constraints on the eclipse depth in the bandpass with the nondetection, as the eclipse phase is effectively fixed in the joint fit by the detection in the other bandpass. We find that the best-fit eclipse phases for HAT-P$5 \mathrm{~b}$, HAT-P-38b, WASP-72b, and WASP-127b are all consistent with the expectation for a circular orbit to within $3 \sigma$. The posterior probability distribution for HAT-P-38b's eclipse phase is bimodal in the version of the fits where the per-point errors are left as free parameters, with one peak within $2 \sigma$ of the predicted phase for a circular orbit and one peak corresponding to a secondary eclipse occurring $\sim 30$ minutes later than expected. The peak corresponding to a circular orbit is the taller of the two peaks in the initial fit, and when we inflate the per-point errors for the visits with significant correlated noise, this secondary peak is further suppressed, indicating that it is likely an artifact of the correlated noise. We therefore present the solution corresponding to the higher peak centered near a phase of 0.5 in Table 3 .

Our best-fit solution for WASP-7b favors an eclipse that occurs $78.8_{-4.2}^{+5.0}$ minutes early, corresponding to an $e \cos (\omega)$ of $-0.0173_{-0.0009}^{+0.0011}$, where $e$ is the orbital eccentricity and $\omega$ is the longitude of periastron. This time offset cannot be due to uncertainties in the planet's ephemeris, as the predicted time of secondary eclipse for a circular orbit has an uncertainty of less than 1 minute (Table 1). It is somewhat surprising that this planet would have an eccentric orbit, as the tidal circularization timescale for this system is predicted to be short $\left(\tau_{\text {circ }}<650 \mathrm{Myr}\right.$; estimated using Equation (2) from Bodenheimer et al. 2001 and a tidal quality factor $Q=10^{6}$ ). This is significantly shorter than the system's 2.4 Gyr estimated age, and WASP-7b does not appear to have an exterior companion capable of maintaining a nonzero eccentricity in the face of ongoing circularization.

We can use the difference in the brightness temperatures between the two bandpasses to ascertain whether we detect changes in a planet's emission spectrum due to the presence of spectral features. For HAT-P-38b and WASP-72b, which have nondetections in one of the two bandpasses, we take a conservative $2 \sigma$ upper limit as the brightness temperature in 


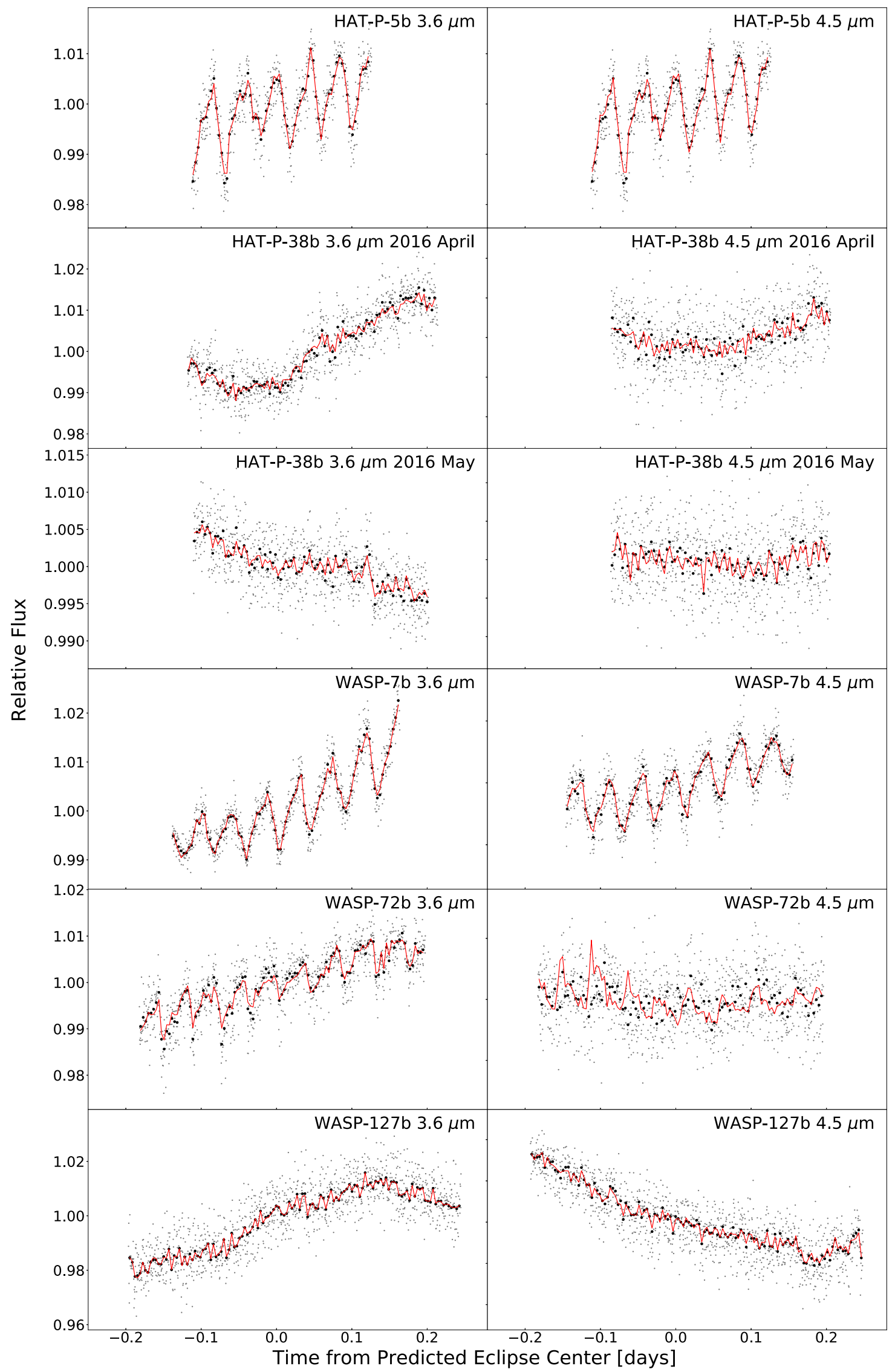

Figure 1. Raw Spitzer photometry for each visit of HAT-P-5b, HAT-P-38b, WASP-7b, WASP-72b, and WASP-127b. The normalized flux binned in 5 minute intervals is shown as black filled circles, and the $30 \mathrm{~s}$ binned flux is shown as gray filled circles. The best-fit instrumental model is overplotted in red. Observations of HAT-P-38b are shown chronologically down each column. 


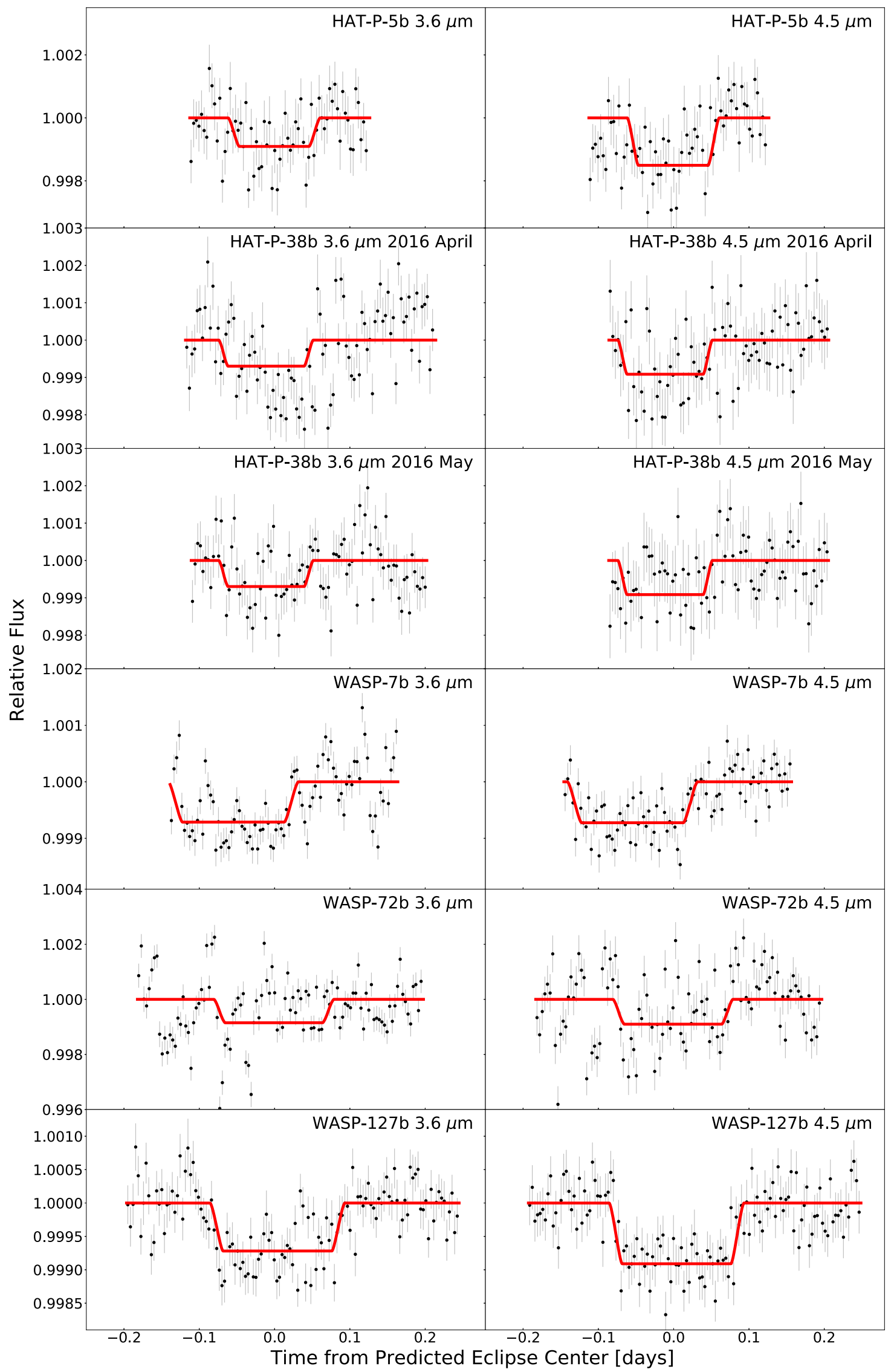

Figure 2. Normalized light curves for each visit of HAT-P-5b, HAT-P-38b, WASP-7b, WASP-72b, and WASP-127b from the simultaneous fits with instrumental effects removed. We show data binned in 5 minute intervals (black filled circles) with error bars corresponding to the scatter in each bin divided by the square root of the number of points in each bin, and we overplot the best-fit secondary eclipse model in red. Observations of HAT-P-38b are shown chronologically down each column. The $2 \sigma$ upper limits for the best-fit eclipse depths of the $4.5 \mu \mathrm{m}$ visits of HAT-P-38b and the $3.6 \mu \mathrm{m}$ visit of WASP-72b are shown. 


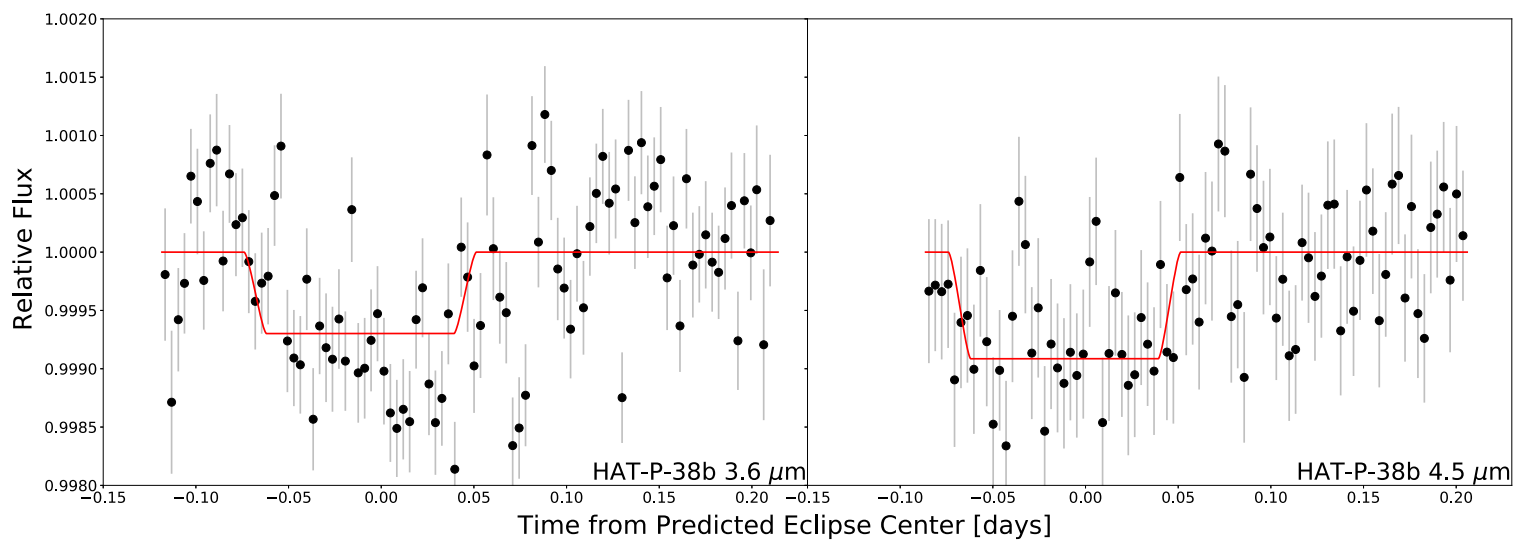

Figure 3. Band-averaged light curves for HAT-P-38b from the simultaneous fits with instrumental effects removed. We show data binned in 5 minute intervals (black filled circles) with error bars corresponding to the scatter in each bin divided by the square root of the number of points in each bin and overplot the best-fit eclipse model in each bandpass for comparison (red lines). The $2 \sigma$ upper limit for the best-fit eclipse depth of the $4.5 \mu \mathrm{m}$ data is shown.

the bandpass with the nondetection. We find that all five planets have spectral shapes that are consistent with those of a blackbody at the $3 \sigma$ level.

\section{Discussion}

We add three of our new secondary eclipse observations to the sample of all Spitzer secondary eclipse measurements from the literature. We include measurements of a subset of the 36 planets (27 of which were new) presented in Garhart et al. (2020; those with detections in both bandpasses measured with a significance greater than $2.5 \sigma ; 31$ planets). We also add 42 planets with published eclipses detected in both bandpasses at better than the $2.5 \sigma$ level (see Table A1 in the Appendix for the full list of included planets). With more than twice the number of planets, we are able to further investigate trends in the spectral shapes of short-period gas giant planets with measured Spitzer secondary eclipses. In order to be able to compare the thermal emission of these planets empirically, we calculate the brightness temperatures for each planet using the reported eclipse depths and planetary and stellar parameters from Southworth (2011; see Table A1 in the Appendix for more details).

\subsection{Trends as a Function of Incident Flux}

In the left panel of Figure 5, we plot the $4.5 \mu \mathrm{m}$ brightness temperature versus the $3.6 \mu \mathrm{m}$ brightness temperature to see if the spectral slopes of these planets deviate from that of a blackbody in a way that correlates with the incident flux. Garhart et al. (2020) found evidence for such a correlation in their study but concluded that the trends they saw were not well matched by commonly used model atmosphere grids. Baxter et al. (2020) used an expanded Spitzer data set to reproduce the trend found by Garhart et al. (2020) and concluded that models including temperature inversions were better able to capture the qualitative shifts in spectral shape as a function of temperature. As shown in the left panel of Figure 5, we also find that the most highly irradiated planets tend to lie above the line (meaning their $4.5 \mu \mathrm{m}$ brightness temperature is higher than their $3.6 \mu \mathrm{m}$ brightness temperature). This is consistent with the predictions of models presented in Lothringer et al. (2018), who showed that planets in this temperature regime should have thermal inversions and additional opacity sources, such as $\mathrm{H}^{-}$, that are not present in cooler atmospheres. At lower temperatures, most of the planets in our sample tend to lie below this line, indicating that their brightness temperatures are relatively high at $3.6 \mu \mathrm{m}$ and low at $4.5 \mu \mathrm{m}$. This is broadly consistent with the predictions of standard atmosphere models (Burrows et al. 1997, 2006; Fortney 2005; Fortney et al. 2008), which suggest that the infrared spectra of these planets should be dominated by water and carbon monoxide absorption bands at these wavelengths. Because carbon monoxide overlaps significantly with the $4.5 \mu \mathrm{m}$ Spitzer band, these models predict that planets in this temperature range should have brightness temperatures that are lower at $4.5 \mu \mathrm{m}$ than at $3.6 \mu \mathrm{m}$ (Garhart et al. 2020). This picture changes for planets with temperatures less than $1000 \mathrm{~K}$ (Wallack et al. 2019), where methane is predicted to be the dominant carbon-bearing molecule.

We can also use the difference between the band-averaged brightness temperatures and the expected equilibrium temperatures for each planet to investigate trends in circulation. As shown in the right panel of Figure 5, the hottest planets in our sample appear to lie significantly above this line, indicating that they have less efficient day-night heat redistribution and low albedos. Indeed, none of these planets lie above the maximum dayside temperature line (calculated from Pass et al. 2019). As the incident flux decreases, planets move closer to the line corresponding to efficient day-night circulation, as predicted by general circulation models (Perez-Becker \& Showman 2013; Komacek \& Showman 2016). This is equivalent to the trend described in Schwartz \& Cowan (2015), where the hottest planets appear to have less efficient redistribution of heat. We quantify the significance of this trend using a Monte Carlo simulation where we generate one realization of the datapoints by sampling from the probability distributions for each point assuming all errors are reasonably well approximated by Gaussian distributions, fitting a line to the resulting realization and repeating this $10^{6}$ times. We find that the slope of the bestfit line differs from the equilibrium temperature expectation by greater than $6 \sigma$ and is qualitatively similar to the trend seen in Schwartz \& Cowan (2015) despite the differences in the stellar models that we use. Schwartz \& Cowan (2015) use a blackbody whereas we use a PHOENIX stellar model from Husser et al. (2013) integrated across the Spitzer bandpass when determining the stellar flux.

We note that a recent study by Baxter et al. (2020) was unable to reproduce this trend in their compilation of Spitzer secondary eclipse data when using a PHOENIX stellar model 


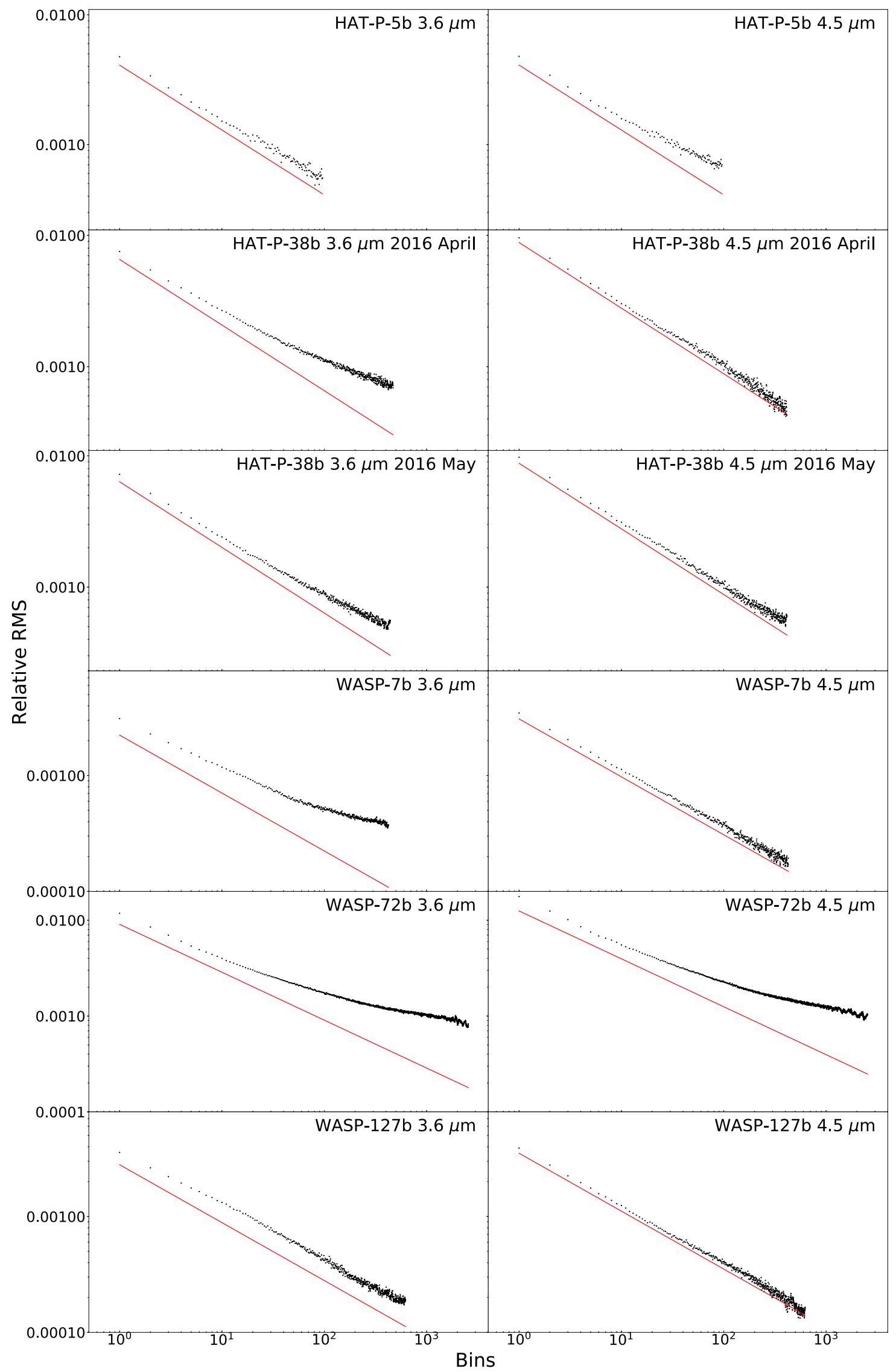

Figure 4. Standard deviation of the residuals as a function of bin size after removing the best-fit instrumental and astrophysical models. The solid lines show the predicted photon noise limit as a function of bin size, which follows a $1 / \sqrt{n}$ scaling. Observations of HAT-P-38b are shown chronologically down each column. 

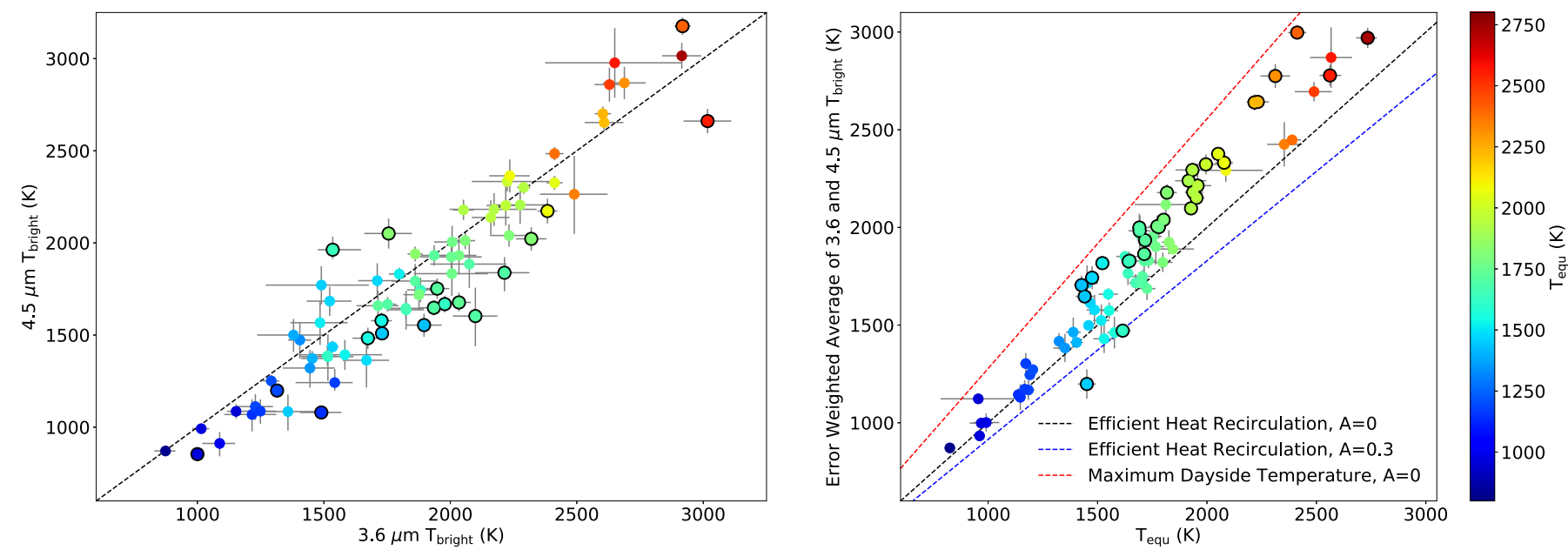

Figure 5. Left: measured $4.5 \mu \mathrm{m}$ brightness temperature vs. $3.6 \mu \mathrm{m}$ brightness temperature for the sample of all planets with published detections in both bands, as well as three new planets from this study. Planets with blackbody-like spectra will lie close to the black dashed line, which corresponds to a 1:1 brightness temperature ratio in the two bands. We highlight points that deviate from this line by greater than $3 \sigma$ (i.e., planets with nonblackbody emission) with black outlines. The colors of the points indicate the predicted equilibrium temperature assuming efficient day-night circulation and zero albedo. Right: error-weighted average of the brightness temperatures measured in the 3.6 and $4.5 \mu \mathrm{m}$ bandpasses vs. the predicted equilibrium temperature. Planets that deviate by more than $3 \sigma$ from the dashed black line (the expected brightness temperature for efficient heat recirculation and zero albedo) are outlined in black.

and integrating across the Spitzer bandpass (although when using a blackbody as in Schwartz \& Cowan (2015), they were able to retrieve the trend). However, this study used the observed brightness temperature at $3.6 \mu \mathrm{m}$ as opposed to the error weighted average of the brightness temperatures in the 3.6 and $4.5 \mu \mathrm{m}$ bandpasses (C. Baxter, email communication). They argued that the presence of $\mathrm{CO}$ absorption in the $4.5 \mu \mathrm{m}$ band makes the observed brightness temperature in this band more sensitive to potential changes in the dayside pressuretemperature profile than the $3.6 \mu \mathrm{m}$ band. We evaluate the significance of the trend in our $3.6 \mu \mathrm{m}$ data using the same Monte Carlo method as before. We find that using only the 3.6 $\mu \mathrm{m}$ data decreases the significance of the trend, but the best-fit line still deviates by $\sim 3 \sigma$ from the line defined by setting the brightness temperature equal to the equilibrium temperature. Although the slope of our best-fit line is consistent with the slope derived from Baxter et al. (2020) to $1 \sigma$, the error on our slope is a factor of $\sim 2$ less, resulting in an increased significance. $^{3}$ The difference in uncertainties between our best-fit slope and that found in Baxter et al. (2020) is likely due to their use of orthogonal distance regression to fit the line and their inclusion of data with large errors (we chose to exclude planets with less than $2.5 \sigma$ detections in either the 3.6 or 4.5 $\mu \mathrm{m}$ bandpass).

Our study also obtains different dayside brightness temperatures than Baxter et al. (2020) for some individual planets. In some cases, this difference is due to the use of different values for the secondary eclipse depths (e.g. using Knutson et al. (2012) instead of Charbonneau et al. (2008) for the eclipse depth of HD $189733 b)$. In other cases, it is due to the use of different values for the planet-star radius ratio and host star properties (effective temperature, metallicity, and gravity). In this study we use the most up-to-date values for the secondary eclipse depths, stellar parameters, and planetary parameters. Specifically, we source our stellar and planetary parameters from TEPCat, a database

\footnotetext{
3 We utilize the $T_{\text {equ }}$ when calculating our slopes with effective temperature, but Baxter et al. (2020) utilize the $T_{\text {irradiation. This choice is inconsequential }}$ when evaluating the statistical significance of the slopes because the difference is just a factor of $1 / \sqrt{2}$, but we state this here for clarity.
}

that seeks to compile the most recent and (where possible) homogeneously derived parameters for each exoplanetary system (Southworth 2008, 2009, 2010, 2011, 2012). Unlike Baxter et al. (2020), we do not do an eccentricity cut on the planets that we include, but instead take the expected equilibrium temperature at the phase of secondary eclipse. We calculate our brightness temperatures using the same method as Baxter et al. (2020; i.e. using a PHOENIX stellar model from Husser et al. (2013) and integrating across each Spitzer bandpass), and we obtain equivalent brightness temperatures when using the same input values.

We conclude that our data provide convincing evidence for a temperature-dependent change in recirculation efficiency. Although this trend might alternatively be interpreted as an increase in the dayside albedos of cooler planets, optical secondary eclipse measurements from Kepler suggest that most planets in this temperature regime have relatively low albedos (Heng \& Demory 2013). There are two planets (WASP-94Ab and WASP-131b) that lie more than $3 \sigma$ below the zero albedo efficient circulation line, suggesting that they may have appreciably higher albedos than the other planets in this sample. Both WASP-94Ab and WASP-131b lie in the same temperature regime as Kepler-7b (Demory et al. 2011, 2013) and HATS-11b (Niraula et al. 2018), both of which have estimated geometric albedos of approximately 0.3 in the optical Kepler bandpass (versus $<0.1$ for most gas giant planets in this temperature regime), likely due to the presence of high-altitude silicate clouds (Demory et al. 2013). If WASP-94Ab and WASP-131b also have optical albedos close to 0.3 , this would be sufficient to explain their lower-than-expected brightness temperatures.

In order to better visualize the changes in the $3.6-4.5 \mu \mathrm{m}$ spectral slope as a function of incident flux, we calculate the difference in brightness temperatures between the two bands and divide by the equilibrium temperature in order to keep the scale of this slope constant across the full temperature range. We plot the resulting scaled slope as a function of the predicted equilibrium temperature assuming zero albedo and efficient circulation in Figure 6. Unsurprisingly, the incident flux 


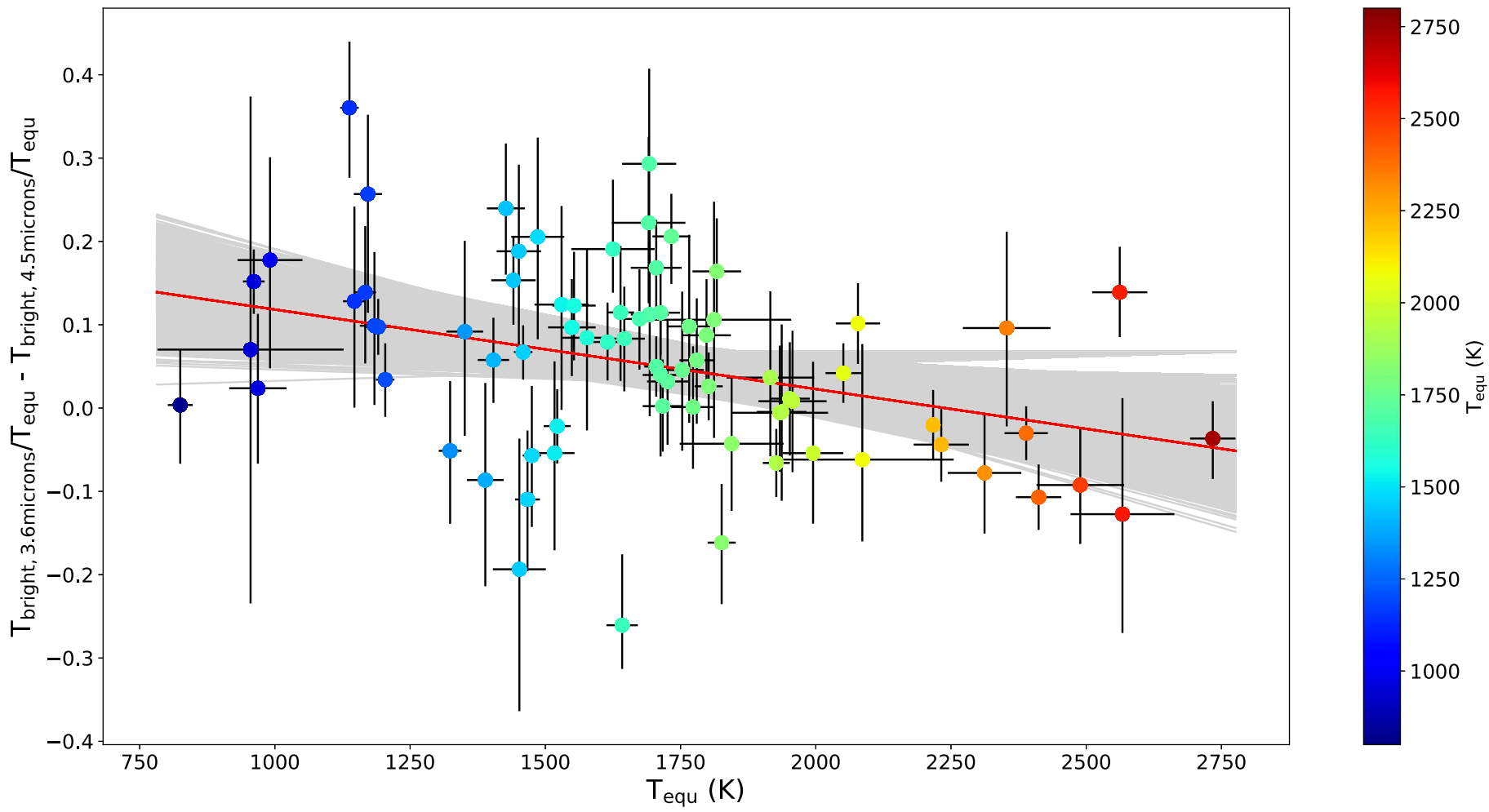

Figure 6. Change in 3.6-4.5 $\mu \mathrm{m}$ slope as a function of equilibrium temperature, where slopes have been normalized by the equilibrium temperature to keep the scale consistent across the full temperature range. The best-fit linear trend is overplotted as a red line with a random sampling of the fit lines from the Monte Carlo, shown as gray lines (see Section 4.1 for a description of the Monte Carlo simulations).

appears to be the primary driver of the observed spectral slope. We use the same Monte Carlo method as before to determine the significance of the trend in Figure 6. We find that negatively sloped lines are preferred at the $3.2 \sigma$ level, suggesting that cooler planets tend to be brighter at $3.6 \mu \mathrm{m}$ and dimmer at $4.5 \mu \mathrm{m}$ than their more highly irradiated counterparts.

\subsection{Trends as a Function of Surface Gravity and Host Star Metallicity}

We next consider whether or not there are additional parameters beyond incident flux that correlate with the observed spectral slopes of these planets. We divide our planet sample into bins according to their predicted equilibrium temperatures and investigate trends in spectral shape within each temperature bin. We first consider whether or not surface gravity can explain some of the observed scatter in the spectral shapes of these planets (see Figure 7).

As before, we evaluate the significance of the trends in spectral slope as a function of surface gravity within each temperature bin using Monte Carlo simulations with $10^{6}$ samples. We find that no temperature bin has a statistically significant slope (see Figure 7).

It is also possible that variations in atmospheric metallicity in this sample of planets might lead to variations in spectral slope. In Wallack et al. (2019), we found a tentative $(\sim 1.9 \sigma)$ correlation between the measured spectral slope of planets with equilibrium temperatures less than $\sim 1000 \mathrm{~K}$ and host stellar metallicity, which we use here as a proxy for planetary atmospheric metallicity. It is reasonable to assume that metalrich stars should have correspondingly metal-rich disks and therefore produce planets with correspondingly metal-enriched atmospheres. However, the range of metallicities spanned by the host stars in our planet sample only vary between -0.41 and +0.43 , corresponding to a relatively small $6.9 \times$ change in bulk disk metallicity. In Wallack et al. (2019), we used a grid of atmosphere models to demonstrate that for cool $(\lesssim 1000 \mathrm{~K})$ planets, these Spitzer observations would only be sensitive to changes in atmospheric metallicity that are 1-2 orders of magnitude larger. Indeed, there are other indications that this picture is not as straightforward as one might hope. For example, Teske et al. (2019) found that there are no statistically significant correlations between host star abundances and bulk planetary metallicity for planets less than $1000 \mathrm{~K}$, further complicating attempts to link stellar metallicity to the resulting planetary atmospheric and bulk metallicities.

We use our new expanded sample to search for trends in stellar metallicity within each temperature bin (Figure 8). Extending the equilibrium temperature bin of the cooler planets to $1300 \mathrm{~K}$, we also recover the relationship between spectral slope and stellar metallicity hinted at in Wallack et al. (2019). In order to determine the significance of the trends in each panel in Figure 8, we again use a Monte Carlo simulation where we sample from the probability distributions for each point. Interestingly, including these slightly warmer $(1000-1300 \mathrm{~K})$ planets in our lowest temperature bin does not greatly decrease the significance of the slope (which is still consistent with a flat line at the $1.8 \sigma$ level), despite using different sources for the stellar metallicities and including warmer planets. Although we would not necessarily expect planets warmer than $1000 \mathrm{~K}$ to have the same metallicitydependent shift in the ratio of methane to carbon monoxide and carbon dioxide that we see in cooler atmospheres, our previous study (as well as Drummond et al. 2018) demonstrated that for planets with $\mathrm{C} / \mathrm{O}$ ratios less than 1 , there is still a detectable 


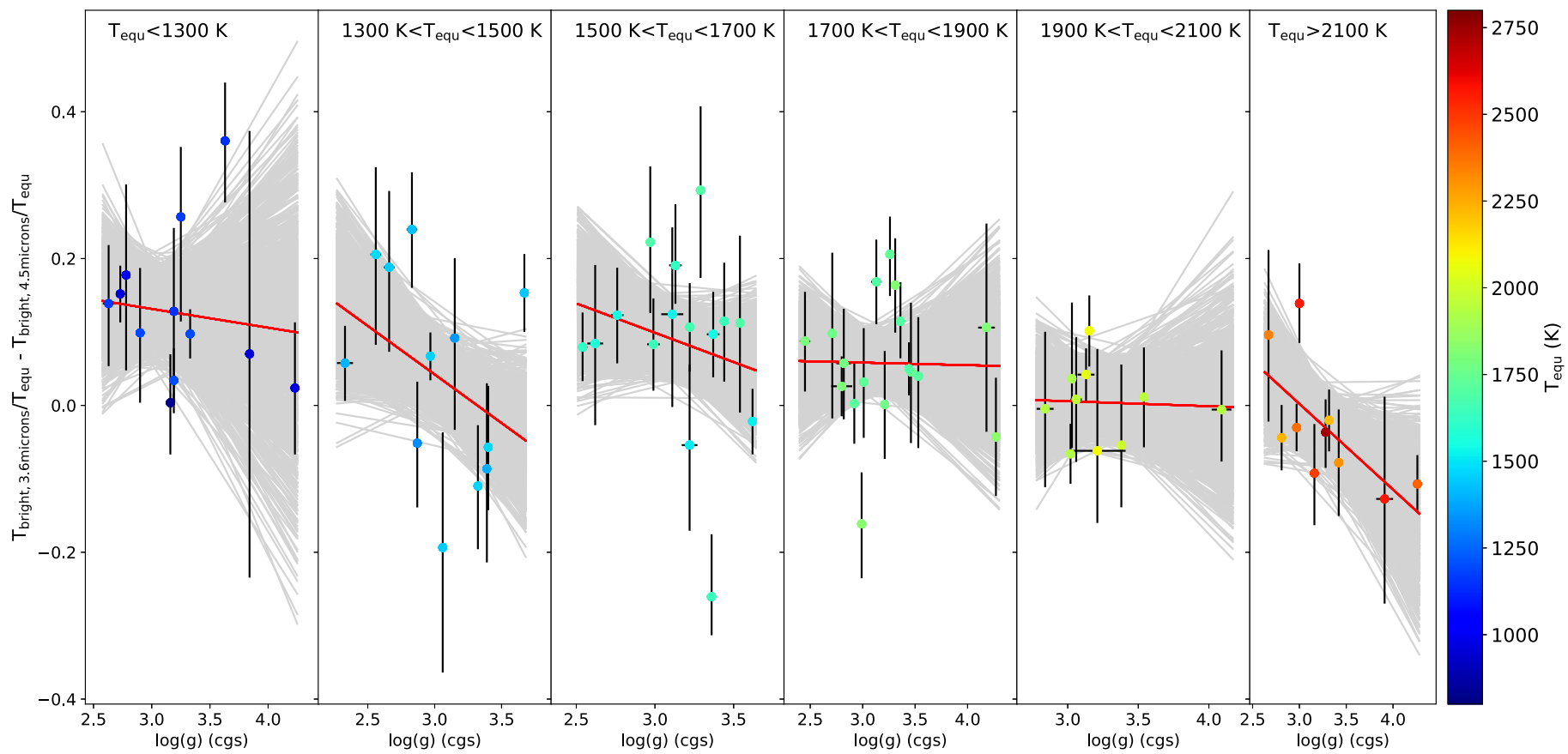

Figure 7. Difference in the two bandpasses, each normalized by equilibrium temperature, vs. planet gravity, divided into temperature bins. The red lines are the best fit from the Monte Carlo simulations, and a random sampling of the fit lines from the Monte Carlo are shown as gray lines (see Section 4.2 for a description of the Monte Carlo simulations).

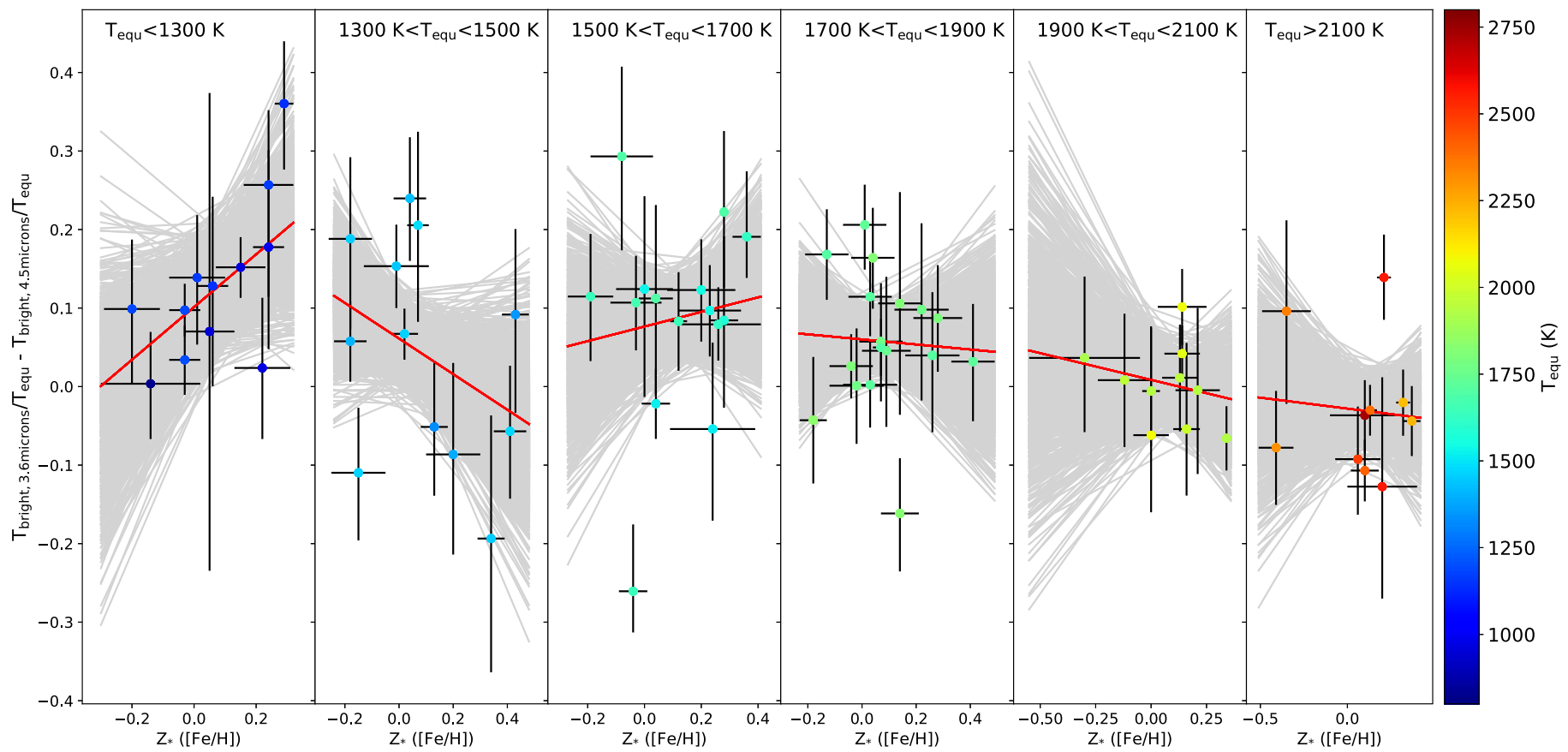

Figure 8. Difference in the two bandpasses, each normalized by equilibrium temperature, vs. stellar metallicity, divided into temperature bins. The red lines are the best fit from the Monte Carlo simulations, and a random sampling of the fit lines from the Monte Carlo are shown as gray lines (see Section 4.2 for a description of the Monte Carlo simulations).

change in the $3.6-4.5 \mu \mathrm{m}$ spectral slope for these slightly warmer planets (see Wallack et al. 2019, Figure 6).

In contrast to this result, we find that negative slopes are preferred for planets in the $1300-1500 \mathrm{~K}$ bin, but our data are still consistent with a positive sloped or flat line at the $1.5 \sigma$ level. It would not be surprising if the effects of metallicity on the spectral slope varied between temperature bins. As discussed above, changes in metallicity can alter the relative abundances of key molecules, including methane, water, carbon monoxide, and carbon dioxide. For planets with highaltitude cloud layers, changes in the metal content of the atmosphere might change the number density, vertical distribution, and average sizes of cloud particles (Morley et al. 2013). In general, we would expect increased cloud opacity at low pressures to suppress the amplitude of molecular features in the planet's dayside emission spectrum, making it 
look more like a blackbody (e.g., Morley et al. 2017) at nearinfrared wavelengths. Indeed, planets with equilibrium temperatures in the 1300-1500 K temperature range are expected to host high-altitude silicate cloud layers, and we might therefore expect this temperature bin to be more sensitive to metallicity-dependent changes in cloud properties at these wavelengths. At mid-infrared wavelengths, on the other hand, we might expect to directly see emission peaks due to the presence of silicate clouds (Richardson et al. 2007).

\section{Conclusions}

We present new secondary eclipse depth measurements in the 3.6 and $4.5 \mu \mathrm{m}$ Spitzer bands for HAT-P-5b, HAT-P-38b, WASP-7b, WASP-72b, and WASP-127b. We find that HATP-5b, HAT-P-38b, WASP-72b, and WASP-127b have secondary eclipse times consistent with the prediction for a circular orbit, but WASP-7b appears to have a modest orbital eccentricity $\left(e \cos (\omega)=-0.0173_{-0.0009}^{+0.0011}\right)$.

We combine these new detections with a sample of 73 planets with published 3.6 and $4.5 \mu \mathrm{m}$ eclipse depths in an effort to better understand trends in the spectral shapes of these planets as a function of irradiation, surface gravity, and host star metallicity. We find that incident flux is the single most important factor for determining the atmospheric chemistry and circulation patterns of short-period gas giant planets. Although we would also expect surface gravity and host star metallicity to play a secondary role, we do not find any compelling evidence for correlations with these parameters in the current sample of Spitzer eclipses. Most planets in our sample with the same incident flux level have broadly similar spectral shapes, but our study also reveals a subset of planets that appear as outliers in these plots. For example, WASP-94Ab and WASP$131 \mathrm{~b}$ appear to be cooler than expected and may have high reflective cloud layers in their dayside atmospheres. Such planets are particularly promising targets for the James Webb Space Telescope (JWST), which is currently scheduled for launch in 2021. The increased aperture size and wavelength coverage of JWST will allow us to obtain invaluable new insights into the atmospheric compositions of gas giant planets.

This work is based on observations made with the Spitzer Space Telescope, which is operated by the Jet Propulsion Laboratory, California Institute of Technology, under a contract with NASA. Support for this work was provided by NASA through an award issued by JPL/Caltech.

\section{Appendix}

We show the full list of planets (both from the literature and from this study) with detected eclipses (at the $2.5 \sigma$ level or better) in both the 3.6 and $4.5 \mu \mathrm{m}$ Spitzer bandpasses in Table A1. Planets shown in bold have new eclipses detected herein.

Table A1

Brightness Temperatures and System Parameters

\begin{tabular}{|c|c|c|c|c|c|c|}
\hline Planet & $T_{\text {equ }}(\mathrm{K})$ & $3.6 T_{\text {bright }}(\mathrm{K})^{\mathrm{a}}$ & $4.5 T_{\text {bright }}(\mathrm{K})^{\mathrm{a}}$ & $\log ($ gravity)(cgs) & {$[\mathrm{Fe} / \mathrm{H}]_{*}$} & References \\
\hline CoRoT-1b & $1916_{-62}^{+81}$ & $2276_{-109}^{+106}$ & $2206_{-102}^{+101}$ & $3.03 \pm 0.03$ & $-0.30 \pm 0.25$ & 1,2 \\
\hline CoRoT-2b & $1522 \pm 25$ & $1798_{-41}^{+40}$ & $1831_{-35}^{+34}$ & $3.62 \pm 0.02$ & $0.04 \pm 0.05$ & 1,2 \\
\hline HAT-P-1b & $1324 \pm 21$ & $1405_{-47}^{+45}$ & $1473_{-101}^{+96}$ & $2.87 \pm 0.01$ & $0.13 \pm 0.05$ & 1,3 \\
\hline HAT-P-2b ${ }^{b}$ & $1812 \pm 143$ & $2232_{-75}^{+74}$ & $2040 \pm 61$ & $4.18 \pm 0.08$ & $0.14 \pm 0.08$ & 1,4 \\
\hline HAT-P-3b & $1172 \pm 26$ & $1543_{-155}^{+71}$ & $1242_{-44}^{+74}$ & $3.25 \pm 0.03$ & $0.24 \pm 0.08$ & 1,5 \\
\hline HAT-P-4b & $1691_{-32}^{+68}$ & $2214_{-116}^{+99}$ & $1838_{-101}^{+85}$ & $2.97_{-0.04}^{+0.02}$ & $0.277 \pm 0.007$ & 1,5 \\
\hline HAT-P-5b & $1517 \pm 37$ & $1485_{-118}^{+109}$ & $1567_{-121}^{+115}$ & $3.22 \pm 0.05$ & $0.24 \pm 0.15$ & 1,6 \\
\hline HAT-P-6b & $1705 \pm 47$ & $1935_{-56}^{+55}$ & $1648 \pm 41$ & $3.13 \pm 0.05$ & $-0.13 \pm 0.08$ & 1,7 \\
\hline HAT-P-7b & $2217 \pm 13$ & $2608_{-77}^{+76}$ & $2653 \pm 49$ & $3.317 \pm 0.007$ & $0.32 \pm 0.04$ & 1,8 \\
\hline HAT-P-8b & $1733 \pm 35$ & $2034_{-68}^{+47}$ & $1677_{-48}^{+53}$ & $3.26 \pm 0.02$ & $0.01 \pm 0.08$ & 1,7 \\
\hline HAT-P-13b & $1726 \pm 38$ & $1714_{-85}^{+81}$ & $1659_{-85}^{+82}$ & $3.01 \pm 0.02$ & $0.41 \pm 0.08$ & 1,9 \\
\hline HAT-P-19b & $991_{-58}^{+60}$ & $1088_{-69}^{+61}$ & $912_{-70}^{+62}$ & $2.78 \pm 0.03$ & $0.24 \pm 0.05$ & 1,10 \\
\hline HAT-P-20b ${ }^{\text {b }}$ & $969 \pm 53$ & $1015_{-35}^{+32}$ & $992 \pm 21$ & $4.23 \pm 0.02$ & $0.22 \pm 0.09$ & 1,11 \\
\hline HAT-P-23b & $1952 \pm 37$ & $2160_{-75}^{+74}$ & $2138_{-94}^{+93}$ & $3.54 \pm 0.03$ & $0.13 \pm 0.08$ & 1,12 \\
\hline HAT-P-30b & $1646 \pm 38$ & $1882_{-53}^{+52}$ & $1745_{-67}^{+66}$ & $2.99 \pm 0.04$ & $0.12 \pm 0.03$ & 1,9 \\
\hline HAT-P-32Ab & $1802 \pm 26$ & $2059_{-40}^{+39}$ & $2012 \pm 46$ & $2.80 \pm 0.10$ & $-0.04 \pm 0.08$ & 1,13 \\
\hline HAT-P-33b & $1780 \pm 32$ & $2034_{-71}^{+69}$ & $1932_{-104}^{+101}$ & $2.82 \pm 0.06$ & $0.07 \pm 0.08$ & 1,9 \\
\hline HAT-P-40b & $1776_{-44}^{+39}$ & $2006_{-150}^{+143}$ & $1833_{-121}^{+117}$ & $2.71 \pm 0.03$ & $0.22 \pm 0.10$ & 1,9 \\
\hline HAT-P-41b & $1937_{-38}^{+46}$ & $2173_{-176}^{+168}$ & $2182_{-90}^{+88}$ & $2.84 \pm 0.06$ & $0.21 \pm 0.10$ & 1,9 \\
\hline HD 149026b & $1625_{-39}^{+77}$ & $1978 \pm 37$ & $1668_{-45}^{+44}$ & $3.13_{-0.04}^{+0.03}$ & $0.36 \pm 0.05$ & 1,14 \\
\hline HD 189733b & $1191 \pm 25$ & $1315 \pm 11$ & $1199 \pm 9$ & $3.33 \pm 0.02$ & $-0.03 \pm 0.05$ & 1,15 \\
\hline HD $209458 b^{c}$ & $1459 \pm 17$ & $1534_{-25}^{+24}$ & $1436_{-33}^{+32}$ & $2.968 \pm 0.004$ & $0.02 \pm 0.05$ & 1,16 \\
\hline KELT-2Ab & $1713_{-29}^{+36}$ & $1948 \pm 48$ & $1752_{-56}^{+55}$ & $3.36 \pm 0.04$ & $0.034 \pm 0.078$ & 1,9 \\
\hline KELT-3b & $1817_{-46}^{+45}$ & $2320_{-61}^{+60}$ & $2022_{-64}^{+63}$ & $3.31 \pm 0.04$ & $0.044_{-0.082}^{+0.080}$ & 1,9 \\
\hline KELT-7b & $2051 \pm 33$ & $2412 \pm 32$ & $2326 \pm 38$ & $3.13 \pm 0.06$ & $0.139_{-0.081}^{+0.075}$ & 1,9 \\
\hline Kepler-5b & $1693_{-29}^{+35}$ & $2075_{-154}^{+147}$ & $1885_{-129}^{+125}$ & $3.54 \pm 0.02$ & $0.04 \pm 0.06$ & 1,17 \\
\hline Kepler-6b & $1452_{-26}^{+49}$ & $1490_{-220}^{+188}$ & $1771_{-105}^{+102}$ & $3.06_{-0.02}^{+0.01}$ & $0.34 \pm 0.05$ & 1,17 \\
\hline Kepler-12b & $1486_{-36}^{+49}$ & $1668_{-94}^{+89}$ & $1363_{-148}^{+135}$ & $2.56 \pm 0.04$ & $0.07 \pm 0.04$ & 1,18 \\
\hline Kepler-13Ab & $2567 \pm 96$ & $2650_{-275}^{+265}$ & $2977_{-190}^{+188}$ & $3.91 \pm 0.09$ & $0.20 \pm 0.20$ & 1,19 \\
\hline
\end{tabular}


Table A1

(Continued)

\begin{tabular}{|c|c|c|c|c|c|c|}
\hline Planet & $T_{\text {equ }}(\mathrm{K})$ & $3.6 T_{\text {bright }}(\mathrm{K})^{\mathrm{a}}$ & $4.5 T_{\text {bright }}(\mathrm{K})^{\mathrm{a}}$ & $\log ($ gravity $)(\operatorname{cgs})$ & {$[\mathrm{Fe} / \mathrm{H}]_{*}$} & References \\
\hline Kepler-17b & $1713_{-67}^{+33}$ & $1861_{-93}^{+89}$ & $1793_{-96}^{+93}$ & $3.53 \pm 0.01$ & $0.26 \pm 0.10$ & 1,20 \\
\hline Qatar-1b & $1389_{-33}^{+34}$ & $1380_{-144}^{+127}$ & $1500_{-91}^{+87}$ & $3.39 \pm 0.01$ & $0.20 \pm 0.10$ & 1,9 \\
\hline TrES-1b & $1147 \pm 21$ & $1216_{-110}^{+96}$ & $1069_{-92}^{+83}$ & $3.19 \pm 0.03$ & $0.06 \pm 0.05$ & 1,21 \\
\hline TrES-2b & $1467 \pm 23$ & $1523_{-91}^{+86}$ & $1684_{-80}^{+78}$ & $3.323 \pm 0.006$ & $-0.15 \pm 0.10$ & 1,22 \\
\hline TrES-3b & $1639 \pm 25$ & $1825_{-73}^{+71}$ & $1637_{-107}^{+103}$ & $3.44 \pm 0.02$ & $-0.19 \pm 0.08$ & 1,23 \\
\hline TrES-4b & $1798 \pm 45$ & $1876_{-62}^{+61}$ & $1719_{-85}^{+83}$ & $2.45 \pm 0.05$ & $0.28 \pm 0.09$ & 1,24 \\
\hline WASP-1b & $1826_{-32}^{+26}$ & $1756_{-96}^{+92}$ & $2051_{-82}^{+81}$ & $2.99{ }_{-0.04}^{+0.03}$ & $0.14 \pm 0.07$ & 1,25 \\
\hline WASP-3b & $1995_{-48}^{+56}$ & $2225_{-140}^{+192}$ & $2333 \pm 54$ & $3.38 \pm 0.03$ & $0.161 \pm 0.063$ & 1,26 \\
\hline WASP-4b & $1674 \pm 29$ & $1824_{-72}^{+70}$ & $1645_{-58}^{+57}$ & $3.221 \pm 0.009$ & $-0.03 \pm 0.09$ & 1,27 \\
\hline WASP-5b & $1753 \pm 40$ & $2004_{-125}^{+120}$ & $1924_{-96}^{+94}$ & $3.46 \pm 0.04$ & $0.09 \pm 0.09$ & 1,28 \\
\hline WASP-6b & $1184 \pm 27$ & $1229_{-77}^{+70}$ & $1112_{-73}^{+68}$ & $2.90 \pm 0.02$ & $-0.20 \pm 0.09$ & 1,10 \\
\hline WASP-7b & $1530 \pm 50$ & $1583_{-161}^{+147}$ & $1393_{-82}^{+80}$ & $3.11 \pm 0.07$ & $0.00 \pm 0.10$ & 1,6 \\
\hline WASP- $8 b^{b}$ & $1138 \pm 17$ & $1490_{-84}^{+79}$ & $1080_{-36}^{+34}$ & $3.63 \pm 0.02$ & $0.29 \pm 0.03$ & 1,29 \\
\hline WASP-10b ${ }^{b}$ & $955_{-173}^{+172}$ & $1153_{-36}^{+34}$ & $1086_{-39}^{+38}$ & $3.84 \pm 0.04$ & $0.05 \pm 0.08$ & 1,10 \\
\hline WASP-12b & $2562_{-48}^{+51}$ & $3017_{-95}^{+94}$ & $2661 \pm 66$ & $3.00 \pm 0.01$ & $0.21 \pm 0.04$ & 1,9 \\
\hline WASP- $14 b^{b, c}$ & $1934_{-77}^{+89}$ & $2290 \pm 26$ & $2301 \pm 35$ & $4.09_{-0.07}^{+0.08}$ & $0.00 \pm 0.04$ & 1,9 \\
\hline WASP-18b & $2412 \pm 42$ & $2918 \pm 32$ & $3176 \pm 48$ & $4.26 \pm 0.02$ & $0.10 \pm 0.08$ & 1,9 \\
\hline WASP- $19 b^{c}$ & $2078 \pm 41$ & $2384 \pm 39$ & $2173_{-68}^{+67}$ & $3.153 \pm 0.005$ & $0.14 \pm 0.11$ & 1,9 \\
\hline WASP-24b & $1773 \pm 40$ & $2007_{-71}^{+70}$ & $2005_{-90}^{+88}$ & $3.21 \pm 0.03$ & $-0.02 \pm 0.10$ & 1,30 \\
\hline WASP-33b & $2734_{-53}^{+42}$ & $2915_{-78}^{+77}$ & $3015 \pm 70$ & $3.28 \pm 0.04$ & $0.10 \pm 0.20$ & 1,14 \\
\hline WASP-39b & $1167 \pm 20$ & $1249_{-66}^{+61}$ & $1087_{-69}^{+64}$ & $2.63 \pm 0.05$ & $0.01 \pm 0.09$ & 1,10 \\
\hline WASP-43b & $1441 \pm 41$ & $1730_{-25}^{+24}$ & $1509_{-32}^{+31}$ & $3.67 \pm 0.01$ & $-0.01 \pm 0.12$ & 1,9 \\
\hline WASP-48b & $1957 \pm 63$ & $2219_{-76}^{+75}$ & $2203_{-109}^{+108}$ & $3.06 \pm 0.04$ & $-0.12 \pm 0.12$ & 1,12 \\
\hline WASP-62b & $1427 \pm 35$ & $1896_{-72}^{+70}$ & $1554_{-64}^{+62}$ & $2.83 \pm 0.04$ & $0.04 \pm 0.06$ & 1,9 \\
\hline WASP-63b & $1577_{-32}^{+46}$ & $1516_{-109}^{+101}$ & $1383_{-131}^{+121}$ & $2.62 \pm 0.05$ & $0.28 \pm 0.05$ & 1,9 \\
\hline WASP-64b & $1692 \pm 50$ & $2099_{-89}^{+87}$ & $1603_{-164}^{+154}$ & $3.29 \pm 0.03$ & $-0.08 \pm 0.11$ & 1,9 \\
\hline WASP-69b & $961 \pm 20$ & $1000 \pm 17$ & $854_{-19}^{+18}$ & $2.73 \pm 0.04$ & $0.15 \pm 0.08$ & 1,31 \\
\hline WASP-74b & $1927 \pm 25$ & $2052 \pm 41$ & $2179 \pm 55$ & $3.02 \pm 0.01$ & $0.34 \pm 0.02$ & 1,9 \\
\hline WASP-76b & $2232 \pm 51$ & $2603 \pm 32$ & $2701 \pm 39$ & $2.81 \pm 0.03$ & $0.366 \pm 0.053$ & 1,9 \\
\hline WASP-77Ab & $1705 \pm 22$ & $1752 \pm 35$ & $1667 \pm 40$ & $3.441 \pm 0.008$ & $0.07 \pm 0.03$ & 1,9 \\
\hline WASP-78b & $2353_{-78}^{+81}$ & $2490_{-136}^{+132}$ & $2264_{-215}^{+208}$ & $2.67 \pm 0.04$ & $-0.35 \pm 0.14$ & 1,9 \\
\hline WASP-79b & $1717_{-34}^{+37}$ & $1936_{-52}^{+51}$ & $1932 \pm 56$ & $2.92 \pm 0.04$ & $0.03 \pm 0.10$ & 1,9 \\
\hline WASP-80b & $825 \pm 23$ & $874_{-44}^{+39}$ & $871 \pm 16$ & $3.16 \pm 0.01$ & $-0.14 \pm 0.16$ & 1,32 \\
\hline WASP-87b & $2312 \pm 68$ & $2688_{-85}^{+84}$ & $2868_{-88}^{+87}$ & $3.42 \pm 0.03$ & $-0.41 \pm 0.10$ & 1,9 \\
\hline WASP-94Ab & $1615_{-32}^{+36}$ & $1514_{-36}^{+35}$ & $1386_{-51}^{+50}$ & $2.54 \pm 0.03$ & $0.26 \pm 0.15$ & 1,9 \\
\hline WASP-97b & $1549 \pm 44$ & $1728_{-42}^{+41}$ & $1578_{-45}^{+44}$ & $3.37 \pm 0.04$ & $0.23 \pm 0.11$ & 1,9 \\
\hline WASP-100b & $2086_{-106}^{+169}$ & $2235_{-81}^{+79}$ & $2364_{-90}^{+89}$ & $3.20_{-0.10}^{+0.20}$ & $0.00 \pm 0.08$ & 1,9 \\
\hline WASP-101b & $1553 \pm 40$ & $1674_{-61}^{+59}$ & $1483_{-58}^{+57}$ & $2.76 \pm 0.04$ & $0.20 \pm 0.12$ & 1,9 \\
\hline WASP- $103 b^{c}$ & $2489_{-88}^{+81}$ & $2629_{-59}^{+58}$ & $2859_{-93}^{+92}$ & $3.16_{-0.03}^{+0.02}$ & $0.06 \pm 0.13$ & 1,9 \\
\hline WASP-104b & $1475 \pm 17$ & $1711_{-76}^{+73}$ & $1795_{-97}^{+95}$ & $3.40 \pm 0.01$ & $0.410 \pm 0.057$ & 1,9 \\
\hline WASP-121b & $2389 \pm 40$ & $2412 \pm 36$ & $2484 \pm 36$ & $2.97 \pm 0.02$ & $0.13 \pm 0.04$ & 1,9 \\
\hline WASP-127b & $1404 \pm 29$ & $1454_{-43}^{+42}$ & $1373_{-41}^{+40}$ & $2.33 \pm 0.06$ & $-0.18 \pm 0.06$ & 1,6 \\
\hline WASP-131b & $1451 \pm 41$ & $1358_{-122}^{+109}$ & $1085_{-103}^{+92}$ & $2.66 \pm 0.04$ & $-0.18 \pm 0.08$ & 1,9 \\
\hline $\mathrm{XO}-1 \mathrm{~b}$ & $1204 \pm 17$ & $1292_{-33}^{+32}$ & $1251_{-34}^{+33}$ & $3.19 \pm 0.01$ & $-0.03 \pm 0.05$ & 1,33 \\
\hline $\mathrm{XO}-2 \mathrm{~b}$ & $1351_{-53}^{+34}$ & $1445_{-107}^{+98}$ & $1321_{-106}^{+98}$ & $3.15 \pm 0.03$ & $0.43 \pm 0.05$ & 1,34 \\
\hline $\mathrm{XO}-3 \mathrm{~b}^{\mathrm{b}}$ & $1844 \pm 96$ & $1861 \pm 30$ & $1940 \pm 40$ & $4.27 \pm 0.03$ & $-0.18 \pm 0.05$ & 1,35 \\
\hline $\mathrm{XO}-4 \mathrm{~b}$ & $1642_{-25}^{+29}$ & $1535_{-59}^{+112}$ & $1963_{-50}^{+71}$ & $3.36 \pm 0.03$ & $-0.04 \pm 0.05$ & 1,7 \\
\hline
\end{tabular}

Notes.

${ }^{a}$ Planetary system parameter values used in computing brightness temperatures from Southworth (2011).

b For this highly eccentric planet (Bonomo et al. 2017), we report the temperature at the planet's separation at secondary eclipse.

${ }^{c}$ We calculate the brightness temperature for this planet using the error-weighted average of the reported eclipses.

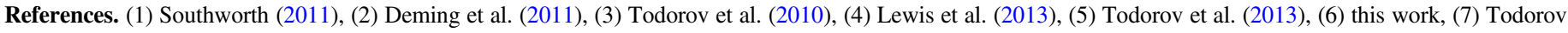

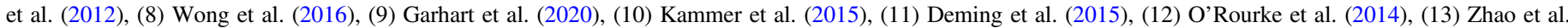

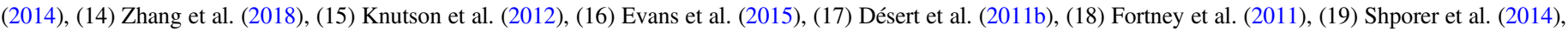

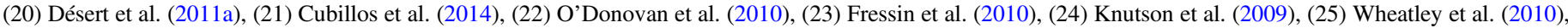

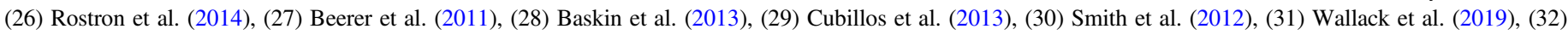
Triaud et al. (2015), (33) Machalek et al. (2008), (34) Machalek et al. (2009), (35) Machalek et al. (2010). 


\section{ORCID iDs}

Nicole L. Wallack (i) https://orcid.org/0000-0003-0354-0187

\section{References}

Albrecht, S., Winn, J. N., Butler, R. P., et al. 2012, ApJ, 744, 189

Bakos, G. Á., Shporer, A., Pál, A., et al. 2007, ApJL, 671, L173

Baskin, N. J., Knutson, H. A., Burrows, A., et al. 2013, ApJ, 773, 124

Baxter, C., Désert, J.-M., Parmentier, V., et al. 2020, A\&A, 639, A36

Beerer, I. M., Knutson, H. A., Burrows, A., et al. 2011, ApJ, 727, 23

Bodenheimer, P., Lin, D. N. C., \& Mardling, R. A. 2001, ApJ, 548, 466

Bonomo, A. S., Desidera, S., Benatti, S., et al. 2017, A\&A, 602, A107

Brogi, M., Line, M., Bean, J., Désert, J.-M., \& Schwarz, H. 2017, ApJL, 839, L2

Burrows, A., Budaj, J., \& Hubeny, I. 2008, ApJ, 678, 1436

Burrows, A., Marley, M., Hubbard, W. B., et al. 1997, ApJ, 491, 856

Burrows, A., Sudarsky, D., \& Hubeny, I. 2006, ApJ, 650, 1140

Charbonneau, D., Allen, L. E., Megeath, S. T., et al. 2005, ApJ, 626, 523

Charbonneau, D., Knutson, H. A., Barman, T., et al. 2008, ApJ, 686, 1341

Chen, G., Pallé, E., Wellbanks, L., et al. 2018, A\&A, 616, A145

Cowan, N. B., \& Agol, E. 2011, ApJ, 726, 82

Cubillos, P., Harrington, J., Madhusudhan, N., et al. 2013, ApJ, 768, 42

Cubillos, P., Harrington, J., Madhusudhan, N., et al. 2014, ApJ, 797, 42

Deming, D., Knutson, H., Agol, E., et al. 2011, ApJ, 726, 95

Deming, D., Knutson, H., Kammer, J., et al. 2015, ApJ, 805, 132

Demory, B.-O., de Wit, J., Lewis, N., et al. 2013, ApJL, 776, L25

Demory, B.-O., Seager, S., Madhusudhan, N., et al. 2011, ApJL, 735, L12

Désert, J.-M., Charbonneau, D., Demory, B.-O., et al. 2011a, ApJS, 197, 14

Désert, J.-M., Charbonneau, D., Fortney, J. J., et al. 2011b, ApJS, 197, 11

Drummond, B., Mayne, N. J., Baraffe, I., et al. 2018, A\&A, 612, A105

Evans, T. M., Aigrain, S., Gibson, N., et al. 2015, MNRAS, 451, 680

Fazio, G. G., Hora, J. L., Allen, L. E., et al. 2004, ApJS, 154, 10

Foreman-Mackey, D., Farr, W. M., \& Sinha, M. 2019, JOSS, 4, 1864

Foreman-Mackey, D., Hogg, D. W., Lang, D., \& Goodman, J. 2013, PASP, 125,306

Fortney, J. J. 2005, MNRAS, 364, 649

Fortney, J. J., Demory, B.-O., Désert, J.-M., et al. 2011, ApJS, 197, 9

Fortney, J. J., Lodders, K., Marley, M. S., \& Freedman, R. S. 2008, ApJ, 678,1419

Fressin, F., Knutson, H. A., Charbonneau, D., et al. 2010, ApJ, 711, 374

Garhart, E., Deming, D., Mandell, A., et al. 2020, AJ, 159, 137

Gillon, M., Anderson, D. R., Collier-Cameron, A., et al. 2013, A\&A, 552, A82

Hellier, C., Anderson, D. R., Gillon, M., et al. 2009, ApJL, 690, L89

Heng, K., \& Demory, B.-O. 2013, ApJ, 777, 100

Heng, K., \& Marley, M. S. 2018, in Handbook of Exoplanets, ed. H. Deeg \& J. Belmonte (Cham: Springer), 102

Husser, T.-O., Wende-von Berg, S., Dreizler, S., et al. 2013, A\&A, 553, A6

Ingalls, J. G., Krick, J. E., Carey, S. J., et al. 2012, Proc. SPIE, 8442, 84421 Y

Kammer, J. A., Knutson, H. A., Line, M. R., et al. 2015, ApJ, 810, 118

Knutson, H. A., Charbonneau, D., Allen, L. E., Burrows, A., \& Megeath, S. T. 2008, ApJ, 673, 526

Knutson, H. A., Charbonneau, D., Burrows, A., O’Donovan, F. T., \& Mandushev, G. 2009, ApJ, 691, 866

Knutson, H. A., Lewis, N., Fortney, J. J., et al. 2012, ApJ, 754, 22

Komacek, T. D., \& Showman, A. P. 2016, ApJ, 821, 16
Kreidberg, L. 2015, PASP, 127, 1161

Kreidberg, L., Line, M. R., Parmentier, V., et al. 2018, AJ, 156, 17

Lam, K. W. F., Faedi, F., Brown, D. J. A., et al. 2017, A\&A, 599, A3

Lanotte, A. A., Gillon, M., Demory, B.-O., et al. 2014, A\&A, 572, A73

Lewis, N. K., Knutson, H. A., Showman, A. P., et al. 2013, ApJ, 766, 95

Lothringer, J. D., Barman, T., \& Koskinen, T. 2018, ApJ, 866, 27

Machalek, P., Greene, T., McCullough, P. R., et al. 2010, ApJ, 711, 111

Machalek, P., McCullough, P. R., Burke, C. J., et al. 2008, ApJ, 684, 1427

Machalek, P., McCullough, P. R., Burrows, A., et al. 2009, ApJ, 701, 514

Mandel, K., \& Agol, E. 2002, ApJ, 580, L171

Morales-Calderón, M., Stauffer, J. R., Kirkpatrick, J. D., et al. 2006, ApJ, 653,1454

Morley, C. V., Fortney, J. J., Kempton, E. M.-R., et al. 2013, ApJ, 775, 33

Morley, C. V., Knutson, H., Line, M., et al. 2017, AJ, 153, 86

Moses, J. I. 2014, RSPTA, 372, 20130073

Moses, J. I., Madhusudhan, N., Visscher, C., \& Freedman, R. S. 2013, ApJ, 763,25

Niraula, P., Redfield, S., de Wit, J., et al. 2018, arXiv:1812.09227

O’Donovan, F. T., Charbonneau, D., Harrington, J., et al. 2010, ApJ, 710, 1551

O'Rourke, J. G., Knutson, H. A., Zhao, M., et al. 2014, ApJ, 781, 109

Pass, E. K., Cowan, N. B., Cubillos, P. E., \& Sklar, J. G. 2019, MNRAS, 489, 941

Perez-Becker, D., \& Showman, A. P. 2013, ApJ, 776, 134

Pont, F., Zucker, S., \& Queloz, D. 2006, MNRAS, 373, 231

Reach, W. T., Megeath, S. T., Cohen, M., et al. 2005, PASP, 117, 978

Richardson, L. J., Deming, D., Horning, K., Seager, S., \& Harrington, J. 2007, Natur, 445, 892

Rostron, J. W., Wheatley, P. J., Anderson, D. R., et al. 2014, MNRAS, 441, 3666

Sato, B., Hartman, J. D., Bakos, G. Á., et al. 2012, PASJ, 64, 97

Schwartz, J. C., \& Cowan, N. B. 2015, MNRAS, 449, 4192

Schwartz, J. C., Kashner, Z., Jovmir, D., \& Cowan, N. B. 2017, ApJ, 850, 154

Shporer, A., O'Rourke, J. G., Knutson, H. A., et al. 2014, ApJ, 788, 92

Smith, A. M. S., Anderson, D. R., Madhusudhan, N., et al. 2012, A\&A, 545, A93

Southworth, J. 2008, MNRAS, 386, 1644

Southworth, J. 2009, MNRAS, 394, 272

Southworth, J. 2010, MNRAS, 408, 1689

Southworth, J. 2011, MNRAS, 417, 2166

Southworth, J. 2012, MNRAS, 426, 1291

Southworth, J., Mancini, L., Maxted, P. F. L., et al. 2012, MNRAS, 422, 3099

Stetson, P. B. 1987, PASP, 99, 191

Teske, J. K., Thorngren, D., Fortney, J. J., Hinkel, N., \& Brewer, J. M. 2019, AJ, 158, 239

Todorov, K., Deming, D., Harrington, J., et al. 2010, ApJ, 708, 498

Todorov, K. O., Deming, D., Knutson, H. A., et al. 2012, ApJ, 746, 111

Todorov, K. O., Deming, D., Knutson, H. A., et al. 2013, ApJ, 770, 102

Torres, G., Winn, J. N., \& Holman, M. J. 2008, ApJ, 677, 1324

Triaud, A. H. M. J., Gillon, M., Ehrenreich, D., et al. 2015, MNRAS, 450, 2279

Wallack, N. L., Knutson, H. A., Morley, C. V., et al. 2019, AJ, 158, 217

Wheatley, P. J., Collier Cameron, A., Harrington, J., et al. 2010, arXiv: 1004.0836

Wong, I., Knutson, H. A., Kataria, T., et al. 2016, ApJ, 823, 122

Zhang, M., Knutson, H. A., Kataria, T., et al. 2018, AJ, 155, 83

Zhao, M., O'Rourke, J. G., Wright, J. T., et al. 2014, ApJ, 796, 115 